\title{
C5a aggravates dysfunction of the articular cartilage and synovial fluid in rats with knee joint immobilization
}

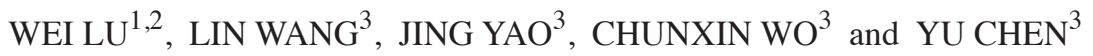 \\ ${ }^{1}$ Department of Anesthesiology, Guizhou Medical University, Guiyang, Guizhou 550004; ${ }^{2}$ Department of Pain Medicine, \\ Cancer Hospital of Guizhou Medical University, Guiyang, Guizhou 550000; ${ }^{3}$ Department of Pain Medicine, \\ Affiliated Hospital of Guizhou Medical University, Guiyang, Guizhou 550004, P.R. China
}

Received September 18, 2017; Accepted March 13, 2018

DOI: $10.3892 / \mathrm{mmr} .2018 .9208$

\begin{abstract}
Degenerative alterations in articular cartilage are involved in the pathogenesis of osteoarthritis. The present study aimed to evaluate the role of complement component 5a (C5a) in osteoarthritic alterations in the articular cartilage and synovialis via a joint immobilization (IM) rat model. Rats were assigned to three groups: Control, IM and IM+anti-C5a antibody (IM+anti-C5a) groups. A terminal deoxynucleotidyl transferase dUTP nick end labeling assay and hematoxylin and eosin staining were used to evaluate the morphological alterations in the articular cartilage and synovialis. Reverse transcription-quantitative polymerase chain reaction (RT-qPCR) analysis, immunohistochemical analysis and western blotting were used to evaluate C5a expression in the articular cartilage and synovialis. An ELISA was used to evaluate C5a-induced alterations in interleukin (IL)-1 $\beta$, IL-17A and tumor necrosis factor (TNF)- $\alpha$ levels in the serum and joint fluid. The results demonstrated that knee joint immobilization induced destruction of knee joint synovial fluid and cartilage in the IM and IM+anti-C5a antibody groups. Immobilization significantly increased the expression levels of C5a in serum and joint fluid in the IM group. Immunohistochemistry, western blotting and RT-qPCR analysis illustrated markedly increased expression of C5a in the IM group. Immobilization markedly increased the IL-1 $\beta$, IL-17A and TNF- $\alpha$ expression levels in the serum and joint fluid in the IM group. Anti-C5a was able to decrease immobilization-induced alterations in morphology and cytokines compared with the IM group. The expression of C5a was increased in synoviocytes and joint cartilage in the IM model. Pro-inflammatory cytokines, including TNF- $\alpha$ and IL-1 $\beta$ were released in the activated synoviocytes via the
\end{abstract}

Correspondence to: Dr Lin Wang, Department of Pain Medicine, Affiliated Hospital of Guizhou Medical University, 28 Guiyi Street, Guiyang, Guizhou 550004, P.R. China

E-mail: guobingbs@sina.com

Key words: complement component 5a, osteoarthritis, cartilage, synoviocytes, joint immobilization induction of $\mathrm{C} 5 \mathrm{a}$, suggesting that $\mathrm{C} 5 \mathrm{a}$ serves an important role in joint inflammatory processes.

\section{Introduction}

Joint immobilization (IM) is a common treatment type in orthopedics, while clinical studies have revealed that joint IM and stress may lead to morphological and biochemical alterations in the articular cartilage. Animal studies have revealed that joint IM induces alterations in the articular cartilage (1-4). A marked decrease in cartilage thickness was observed in animals with chronic joint IM (5-7). Alterations in proteoglycan synthesis, fibrofatty proliferation and chondromalacia were additionally reported in animals with joint immobilization (1-13). Similar morphological alterations in the articular cartilage were observed in patients with joint fixation treatment, which may in turn induce osteoarthritis (OA). Thus, investigation into the underlying mechanism of articular cartilage degeneration induced by joint immobilization remains necessary to develop strategies for the prevention and treatment of complications of joint immobilization, including OA (8-11).

$\mathrm{OA}$ is characterized by articular cartilage degeneration, osteophyte formation and a narrowed joint space (12). Joint cartilage is involved in the development of OA (13). A number of studies have suggested that increased pro-inflammatory cytokines are critical to the development of OA (14). Infiltration and activation of immune cells (including macrophages, mast cells and T cells) in the joints induce synovitis and damage the joint cartilage (14-16). These results indicate that studying the role of immune factors associated with synovitis assists in better understanding the clinical features of joint IM induced by OA.

Complement component C5a (C5a) is an important pro-inflammatory peptide, and is involved in complement activation, membrane attack complex formation, chemotaxis of immune cells and allergic responses $(17,18)$. C5a is generated by cleavage of complement component $\mathrm{C} 5$ by protease C5-convertase $(17,18)$. A number of studies have assessed the role of $\mathrm{C5}$ a and its associated proteins in inflammatory joint diseases. The interaction between $\mathrm{C} 5 \mathrm{a}$ and $\mathrm{C} 5 \mathrm{aR}$ induces the release of TNF- $\alpha$ in activated synoviocytes $(19,20)$, suggesting that the $\mathrm{C} 5 \mathrm{a} / \mathrm{C} 5 \mathrm{aR}$ system may serve an important role in the joint inflammation process. Although the complement 
system is implicated in the inflammatory processes of arthritic diseases, direct interaction between $\mathrm{C} 5 \mathrm{a}$ and joint IM induced by OA has not been demonstrated.

In the present study, it was hypothesized that C5a may be involved in the pathogenesis of joint IM-induced OA, and inhibition of C5a may act as a novel therapeutic target for the prevention of OA. Therefore, to verify this hypothesis, the role of C5a in the development of OA was studied in a chronic knee fixation animal model.

\section{Materials and methods}

Animal model and experiments design. A total of 72 adult male Sprague-Dawley rats, (380-400 g; 14 weeks) were purchased from the Guangdong Medical Laboratory Animal Center (Foshan, China) were used in the present study. The rats were randomly assigned into three groups: Control group ( $n=24)$, immobilization group (IM group, $n=24)$, and immobilization+anti-C5a antibody group (IM+anti-C5a group, $n=24)$. The rats in the control group did not undergo any treatment, while rats in the IM and IM+anti-C5a groups underwent the following procedure: A degreasing cotton patch was placed in the popliteal fossa of unilateral $(50 \%$ right and $50 \%$ left in each group) knee joints to immobilize the joint at $180^{\circ}$ and fixed with a plaster bandage (Anji Wande Medical Products Co., Ltd., Anji, China) for 6 weeks. Saline (1 ml) or anti-C5a antibody $(0.1 \mu \mathrm{g} / \mathrm{ml}$; Wuhan Boster Biological Technology, Ltd., Wuhan, China; cat. no. PB0184) were given every week for 6 weeks. A total of four rats/group for 6 weeks were sacrificed at the end of each week for the subsequent assays. All procedures were approved by the Guizhou Medical University (Guiyang, China) Animal Care and Use Committees and were in accordance with the National Institutes of Health Guide (1996) for the care and use of laboratory animals (21).

\section{Tissue preparation}

Cartilage dissection. The femur was truncated at $5 \mathrm{~cm}$ above the femoral condyle, and tibia was truncated at $3 \mathrm{~mm}$ below the tibial plateau. Soft tissue such as muscle, fat, periosteum surrounding knee joint were removed carefully. Patellar ligament, medial and lateral collateral ligament and anterior cruciate ligament was cut off, and the aponeurotic laminae on both sides were cut open. The patella is flipped down. The knee is opened and the articular cartilage is cut with a $15 \#$ scalpel blade. The cartilage on each joint surface is cut off. Do not accidentally bring the subchondral bone and periosteum.

Synovectomy. Cut the skin longitudinally along the median knee joint until an area of about $3 \times 3 \mathrm{~cm}$ centered on the knee joint is exposed. Lift the patella with a tweezers. femur was truncated at $0.13 \sim 0.14 \mathrm{~cm}$ above the upper edge of patella. Split down to the tibia along both sides of the patella. After the knee joint cavity was opened, a layer of smooth and shiny yellowish synovial tissue adhered to the lower edge of the patella can be seen.

Terminal deoxynucleotidyl transferase dUTP nick end labeling (TUNEL) assay and hematoxylin and eosin (H\&E) staining. The specimens were fixed with $4 \%$ paraformaldehyde in $0.1 \mathrm{M}$ phosphate buffer, at $\mathrm{pH} 7.4$ overnight at $4^{\circ} \mathrm{C}$. The tissues were dehydrated through a series graded ethanol baths $(100,95,90$, 80 and $70 \%$ ), and the specimens were embedded in paraffin. The embedded tissue was cut into $4 \mu \mathrm{m}$ sections. The center of the scanned region was set for histological evaluation.

TUNEL staining of fractured double-stranded DNA in apoptotic cells was performed. Paraffin sections $(4 \mu \mathrm{m})$ of synovial and cartilage tissues were used for the evaluation of apoptosis by TUNEL staining. The In Situ Cell Death Detection kit-POD (Roche Diagnostics, Basel, Switzerland; cat. no. 11684817910) was used to determine the number if apoptotic cells, according to the manufacturer's protocol. Counting of TUNEL-positive cells was conducted on x200-magnified sections for each group. A H\&E Staining kit (Shanghai Yeasen Biotechnology Co., Ltd., Shanghai, China, cat. no. 60524ES60) was used for staining the cardiac muscle, according to the manufacturer's protocols. Briefly, slides were stained for 5 min with hematoxylin, washed with diluted water three to five times at room temperature and subsequently stained with eosin solution for $20 \mathrm{sec}$ at room temperature.

Reverse transcription-quantitative polymerase chain reaction $(R T-q P C R)$. Total RNA was extracted from the synovial fluid and cartilage tissue by using TRIzol reagent (Thermo Fisher Scientific, Inc., Waltham, MA, USA). cDNA was synthesized using $1 \mu \mathrm{g}$ total RNA via an RT kit (DBI Bioscience, Newark, DE, USA). The RNA solution was incubated at $65^{\circ} \mathrm{C}$ for $5 \mathrm{~min}$, and the temperature protocol for the reverse transcription reaction was $37^{\circ} \mathrm{C}$ for $15 \mathrm{~min}$, followed by $98^{\circ} \mathrm{C}$ for $5 \mathrm{~min}$. The primers used in qPCR were as follows: C5a sense, $5^{\prime}-\mathrm{CTC}$ ACCTTCCTCACCAATGC-3' and antisense, 5'-GCTTGT TCTTCCACTTTCTGAT-3'; GAPDH sense, 5'-CCTCGTCTC ATAGACAAGATGGT-3' and antisense, 5'-GGGTAGAGT CATACTGGAACATG-3'. SYBR-Green qPCR master Mix (DBI Bioscience) was used for qPCR amplification. Cycling conditions included denaturation at $95^{\circ} \mathrm{C}$ for $2 \mathrm{~min}$ followed by 40 cycles of annealing at $94^{\circ} \mathrm{C}$ for $20 \mathrm{sec}$ and extension at $58^{\circ} \mathrm{C}$ for $20 \mathrm{sec}$. On the basis of exponential amplification of the target gene relative to the housekeeping gene as an internal reference, the quantity of amplified molecules at the quantification cycle was given by $2^{-\Delta \Delta \mathrm{Cq}}(22)$.

ELISA analysis. Expression levels of IL-1 $\beta$, C5a, TNF- $\alpha$ and IL-17A in the synovial fluid and cartilage tissue were measured using an antigen-based sandwich ELISA. The Rat IL-1 $\beta$ ELISA kit (cat. no. E-EL-R0012c), Rat C5a ELISA kit (cat. no. E-EL-R0257c), Rat TNF- $\alpha$ ELISA kit (cat. no. E-EL-R0019c) and Rat IL-17A ELISA Kit (cat. no. E-EL-R0566c; all Elabscience, Houston, TX, USA) were used. The absorbance was measured at $450 \mathrm{~nm}$ using a microplate spectrophotometer (Multiskan GO; Thermo Fisher Scientific, Inc.).

Western blot analysis. A total of $40 \mathrm{mg}$ synovial fluid and cartilage tissue was placed in $120 \mu \mathrm{l}$ radioimmunoprecipitation assay (RIPA) lysis buffer (Beyotime Institute of Biotechnology, Shanghai, China) with phosphatase and protease inhibitors (BIOSS, Beijing, China) for $2 \mathrm{~h}$ at room temperature. Protein concentration was determined with bicinchoninic acid protein assay. Total protein $(20 \mu \mathrm{l})$ was separated on $12 \%$ Tris-glycine polyacrylamide gels. Samples were subsequently transferred 


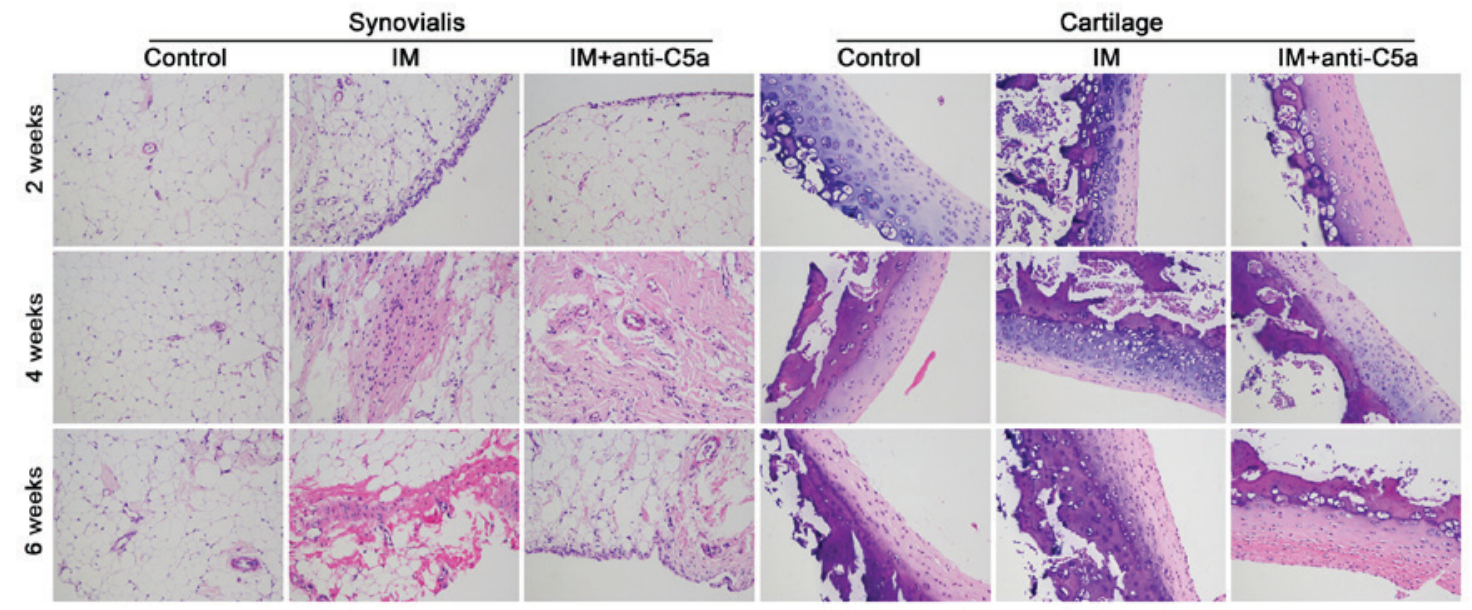

Figure 1. Joint IM induces destruction of the synovialis and cartilage in rats. Hematoxylin and eosin staining was performed. Magnification, x200. IM, immobilization; C5a, complement component 5a.

onto polyvinylidene fluoride membranes (Pall Life Sciences, Port Washington, NY, USA). Membranes were incubated in blocking buffer (cat. no. P0023B; Beyotime Institute of Biotechnology) for $8 \mathrm{~h}$ at $4^{\circ} \mathrm{C}$, and subsequently incubated with rabbit anti-C5a (cat. no. ab11876; 1:2,000), rabbit anti-IL-1 $\beta$ (cat. no. ab200478; 1:2,000), rabbit anti-TNF- $\alpha$ (cat. no. ab9755; 1:2,000; all Abcam, Cambridge, UK;), rabbit anti-IL-17A (cat. no. SAB3701458-100UG; 1:2,000; Sigma-Aldrich; Merck KGaA, Darmstadt, Germany), and rabbit anti-GAPDH (cat. no. KC-5G5; 1:5,000; Kangchen BioTech Co., Ltd., Shanghai, China) for $8 \mathrm{~h}$ at $4^{\circ} \mathrm{C}$. Horseradish peroxidase (HRP) goat anti-rabbit immunoglobulin $\mathrm{G}$ secondary antibody (cat. no. BA1054; 1:5,000; Wuhan Boster Biological Technology, Ltd.) was added for $1 \mathrm{~h}$ at $25^{\circ} \mathrm{C}$. Enhanced chemiluminescence substrate was used for protein visualization (Pierce; Thermo Fisher Scientific, Inc.). The specific protein band intensities were quantified by Image-Pro Plus 6.0 (Media Cybernetics, Inc, Rockville, MD, USA).

Immunohistochemical analysis. Slices were subjected to treatment using 3\% hydrogen peroxide for $10 \mathrm{~min}$ at room temperature for endogenous peroxidase inactivation. The sections were subsequently blocked using $1 \%$ bovine serum albumin (Gibco; Thermo Fisher Scientific, Inc.) at room temperature for $4 \mathrm{~h}$ to reduce non-specific staining. For immunohistochemical staining, the sections were incubated with primary antibodies against C5a (cat. no. ab11876; 1:1,000; Abcam) for $8 \mathrm{~h}$ at $4^{\circ} \mathrm{C}$. After washing with PBS, the slides were incubated with goat anti-rabbit IgG biotinylated-modified secondary antibody (cat. no. A27035; 1:2,000; Thermo Fisher Scientific, Inc.), and conjugated HRP-labeled streptavidin (cat. no. K0609; 1:2,000; Dako; Agilent Technologies, Inc., Santa Clara, CA, USA) was added. Following staining, three high-magnification fields (x400) were randomly selected in each slide, and the images were recorded using an inverted light microscope (Zeiss AG, Oberkochen, Germany).

Masson staining. Paraffin sections underwent gradient dehydration by incubating the slices in $100 \%$ xylene and a graded ethanol series (100, 95, 90, 80 and 70\%). Masson staining of Weigert's iron was performed with a Masson Trichome Staining kit, according to the manufacturer's protocol (cat no. M029; Shanghai Gefan Biotechnology Co., Ltd., Shanghai, China) for $10 \mathrm{~min}$ at room temperature. The stained slices underwent Ponceau $\mathrm{S}$ staining for $10 \mathrm{~min}$ at room temperature, and treatment using phosphomolybdic acid for $5 \mathrm{~min}$ at room temperature. Aniline blue staining was performed, and the slices subsequently underwent differentiation in $1 \%$ glacial acetic acid for $1 \mathrm{~min}$. Slices were treated using dehydrated alcohol (95 and 100\%) and 100\% xylene. Slides were subsequently viewed under an inverted light microscope.

Statistical analysis. All statistical analyses were performed with SPSS 19.0 software (IBM Corp., Armonk, NY, USA). The data are presented as mean \pm standard error of the mean from three separate experiments. Statistical significance was determined by paired or unpaired Student's t-test and one-way analysis of variance followed by Tukey's post-hoc test in cases of standardized expression data. $\mathrm{P}<0.05$ was considered to indicate a statistically significant difference.

\section{Results}

Knee joint IM induces marked destruction of the synovial fluid and cartilage. Morphological analysis was conducted to investigate the effect of knee joint IM on the synovial fluid and cartilage. H\&E and Masson staining were used. As presented in Fig. 1, prolonged knee joint IM resulted in aggravation of the destruction of the synovial fluid and cartilage in the IM group, while anti-C5a antibody treatment reversed these alterations. Masson staining revealed marked fibroplasia in the synovial membrane and cartilage (Fig. 2). TUNEL staining demonstrated apoptosis in the synovial membrane and cartilage. As presented in Fig. 3, the staining intensity in the IM group was increased with prolonged treatment, indicating increased cellular apoptosis in the synovial membrane and cartilage, and treatment with anti-C5a mitigated these alterations.

Increased C5a and pro-inflammatory cytokines in the joint cavity fluid and serum in rats with knee joint IM. The expression levels of C5a, IL-1 $\beta$, TNF- $\alpha$ and IL-17A in the serum (Fig. 4A) and joint cavity fluid (Fig. 4B) were evaluated 


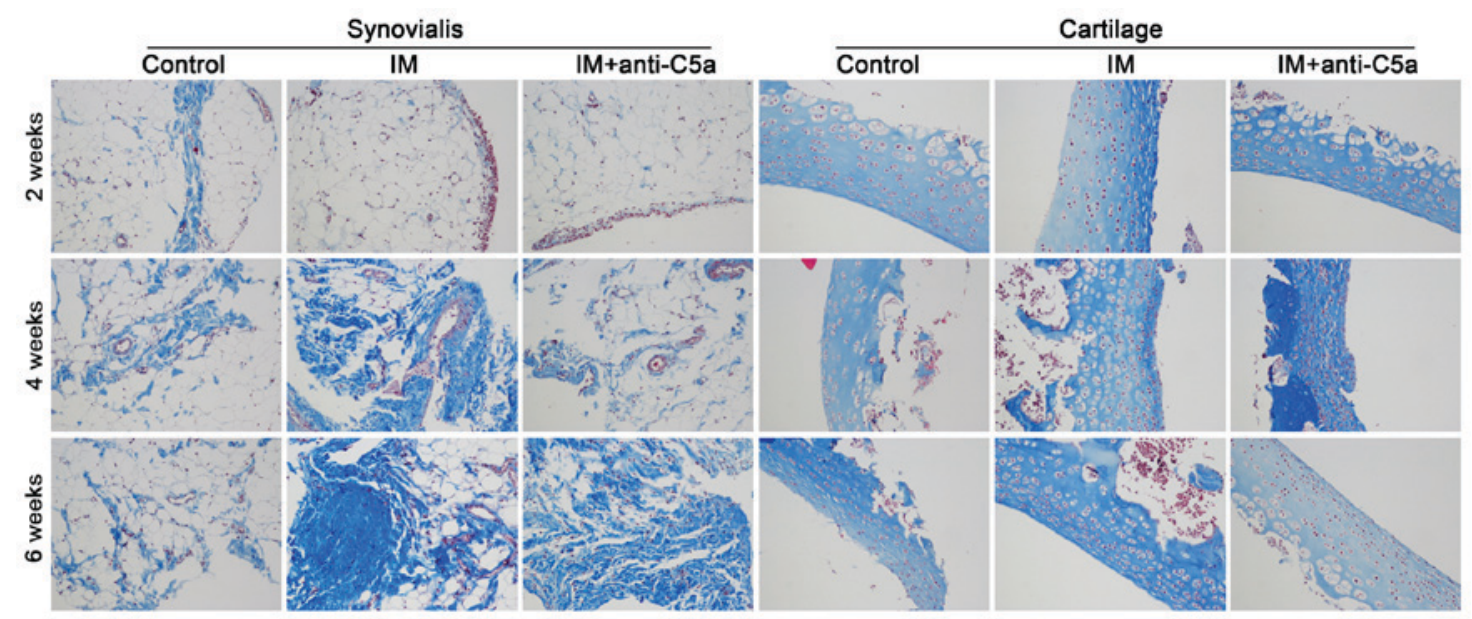

Figure 2. Effects of joint IM on collagen accumulation in the synovialis and cartilage. Masson staining was performed. Magnification, x200. IM, immobilization; C5a, complement component 5a.

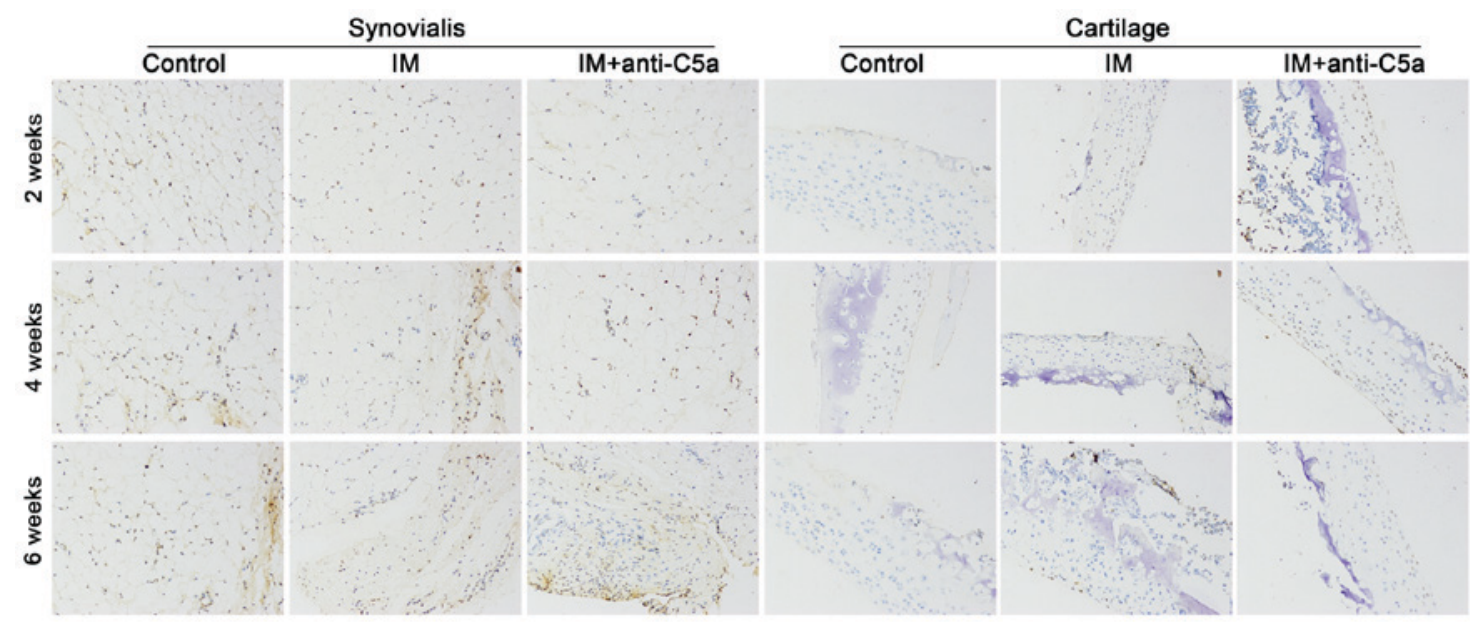

Figure 3. Apoptosis of synovial and cartilage cells is observed in rats with joint IM. Terminal deoxynucleotidyl transferase dUTP nick end labeling was performed. Magnification, x200. IM, immobilization; C5a, complement component 5a.

using ELISA. The results revealed that following 1 week of knee joint IM, the expression levels of C5a, IL-1 $\beta$, TNF- $\alpha$ and IL-17A in the joint cavity fluid and serum were significantly increased compared with those in the control group (Fig. 4). These results indicated that knee joint IM significantly induced inflammation in the knee joint. With prolonged knee joint IM, the expression levels of C5a, IL-1 $\beta$, IL-17A and TNF- $\alpha$ in the IM group were gradually increased during the experiment compared with the control and IM+anti-C5a groups. However, the levels of C5a, IL-1 $\beta$, IL-17A and TNF- $\alpha$ in the joint cavity fluid of in IM+anti-C5a group were lower compared with those in the IM group.

Increased levels of C5a in the synovial membrane and cartilage in rats with knee joint immobilization. An RT-qPCR assay, western blot analysis and immunohistochemistry were performed to assess the expression of $\mathrm{C} 5 \mathrm{a}$ in the synovialis and cartilage. The expression patterns of C5a in the synovial fluid and cartilage in the rat knee joint following IM with different treatments were evaluated by immunohistochemistry. The results revealed that the expression of $\mathrm{C} 5 \mathrm{a}$ was significantly decreased in the IM+anti-C5a group compared with the IM group (Fig. 5). In addition, the expression of C5a mRNA was increased following 1 week of knee joint IM in the IM and IM+anti-C5a groups in the synovial fluid (Fig. 6A, left) and cartilage (Fig. 6A, right), and western blotting demonstrated similar results in the synovial fluid (Fig. 6B) and cartilage (Fig. 6C). These results confirmed the findings that increased levels of C5a may serve an important role in the development of chronic inflammation in the immobilized knee joint, and dysfunction of the synovial fluid and cartilage induced by OA.

\section{Discussion}

The present study investigated the role of C5a in the development of OA in rats with joint IM. A knee cartilage injury was induced that mimicked OA by fixing the rat knee joint at an angle of $180^{\circ}$. In rats with knee joint IM, destruction of the knee joint synovial fluid and cartilage was observed. The expression levels of C5a in the serum and joint fluid were assessed, and it was demonstrated that IM significantly increased the expression levels of C5a. Immunohistochemistry, western blotting 

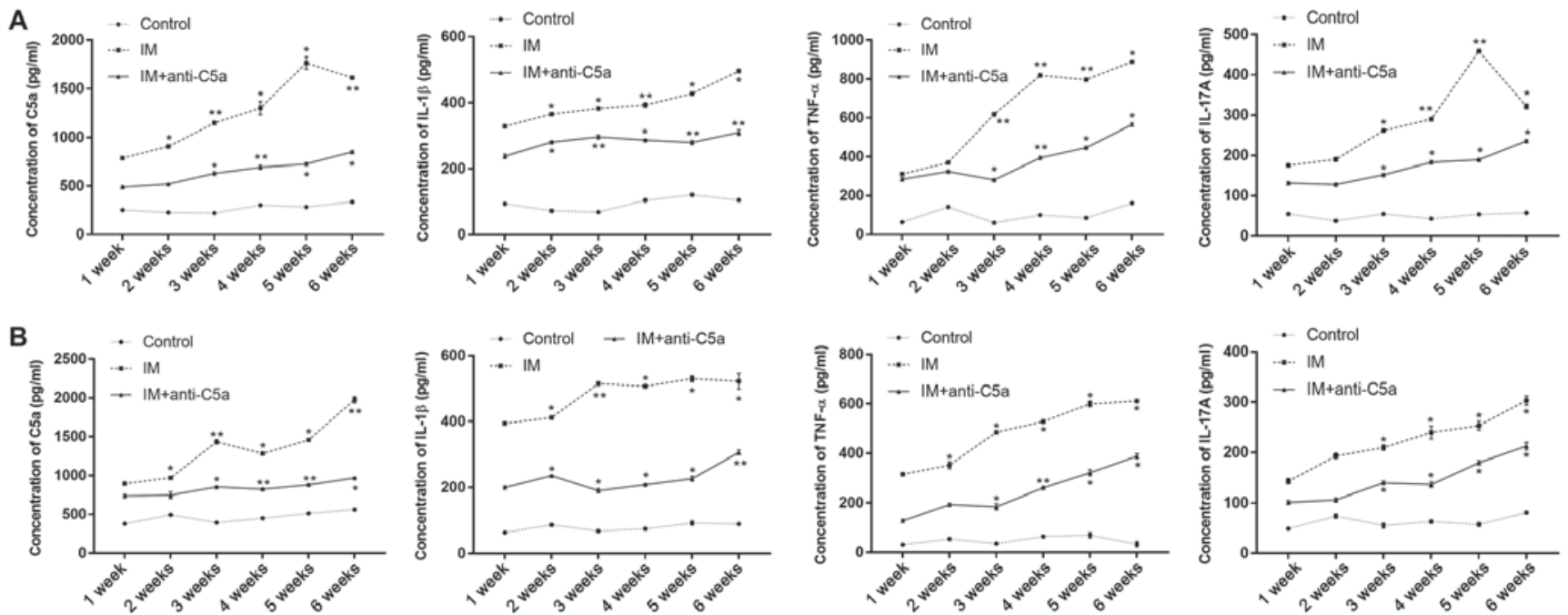

Figure 4. Effects of joint IM on serum C5a and pro-inflammatory cytokine expression levels. (A) Expression levels of C5a, IL-1 $\beta$, TNF- $\alpha$ and IL-17A in the serum; (B) expression levels of C5a, IL-1 $\beta$, TNF- $\alpha$ and IL-17A in joint cavity fluid. The graphs present the mean \pm SEM. ${ }^{*} \mathrm{P}<0.05,{ }^{* *} \mathrm{P}<0.01 \mathrm{vs}$. control group at the same time point. The data are presented as the average of triplicate values. Error bars represent the SEM. IM, immobilization; C5a, complement component 5a; SEM, standard error of the mean; IL, interleukin; TNF- $\alpha$, tumor necrosis factor- $\alpha$.

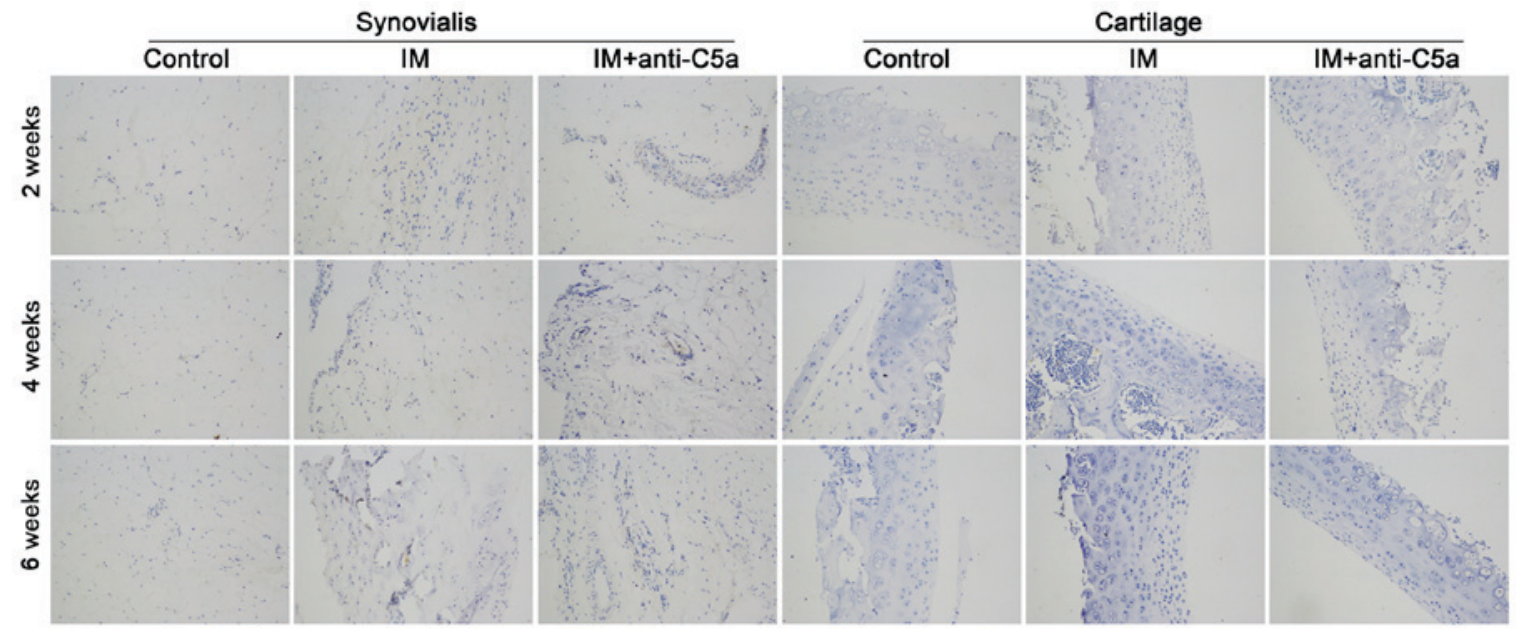

Figure 5. Joint IM increases the expression of C5a in the synovialis and cartilage. Immunohistochemical staining of C5a and Masson staining was performed. Magnification, x200. IM, immobilization; C5a, complement component 5a.

and RT-qPCR analysis demonstrated a significant increase in the expression of C5a. The application of anti-C5a antibody was able to mitigate the IM-induced alterations in morphology and cytokine expression in the knee joint synovial fluid and cartilage. Joint IM significantly increased the expression levels of IL-1 $\beta$, IL-17A and TNF- $\alpha$ in the serum and joint fluid.

Dysfunction of articular cartilage remains the characteristic pathology of OA $(12,13)$. Articular cartilage serves an extremely important role in maintaining the physiological function of the knee joint $(6,13,14)$. Alterations in the immune system in the elderly population are associated with the development of primary OA. Decreased T and B cell function, and elevated pro-inflammatory cytokine expression was observed in patients with OA (23-26). These studies indicated that abnormal immune responses may contribute to the development of cartilage.

The present study additionally demonstrated that rats with knee joint IM exhibited destruction of the knee joint synovial fluid and cartilage, increasing the levels of C5a in the serum and joint fluid significantly. A central role for complement in OA has been determined by a series of studies (27-31). C5a is a highly inflammatory peptide that acts as a chemotactic agent, and additionally serves an important role in innate immunity $(17,18)$. C5a was able to increase the expression of adhesion molecules and trigger mast cell degranulation, releasing proinflammatory molecules, including histamine and TNF- $\alpha(32,33)$. C5a is an effective chemoattractant, and initiates the accumulation of complement and phagocytic cells at sites of infection or the recruitment of antigen-presenting cells to the lymph nodes $(34,35)$. The complement system is activated in the synovial fluid of subjects with knee injury and OA (30). Increased expression of C5aR is observed in synoviocytes in joint inflammatory diseases $(16,19,35)$. C5a/C5aR interaction induces the release of TNF- $\alpha$ in activated synoviocytes, promoting joint inflammatory processes $(16,19,35)$. The present study revealed the role of C5a in the development of 
A
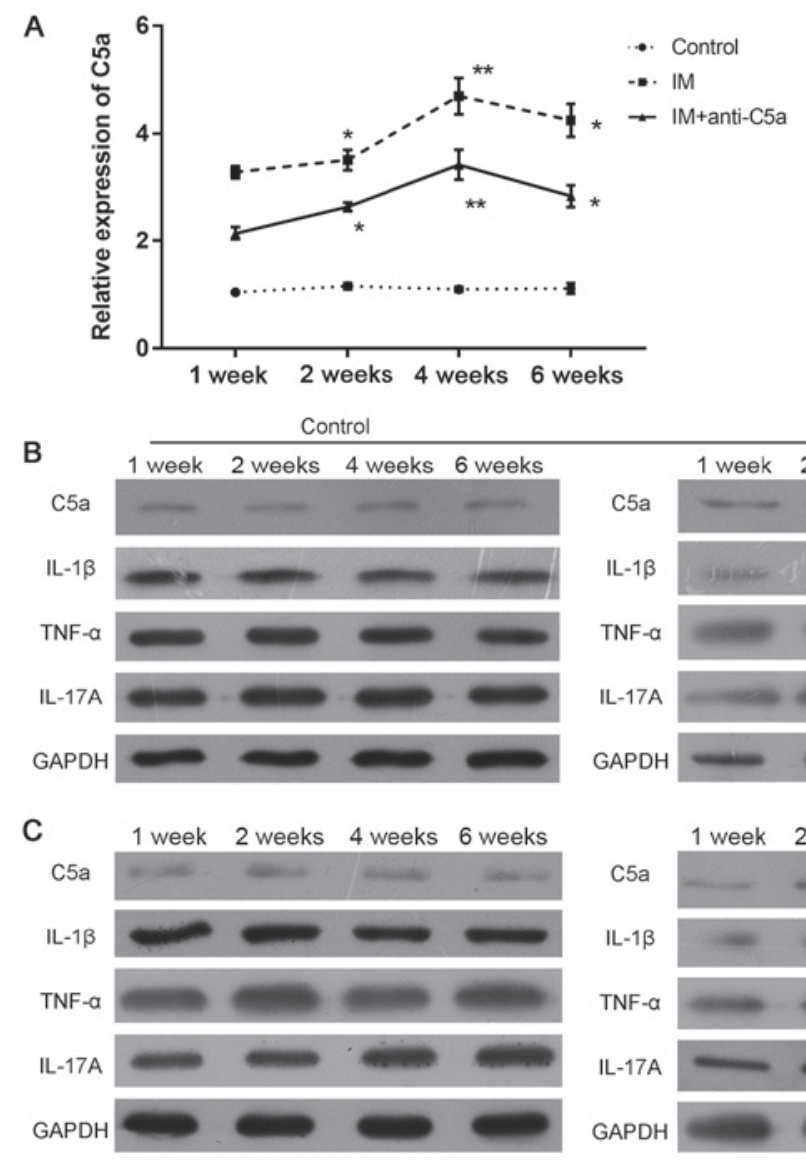
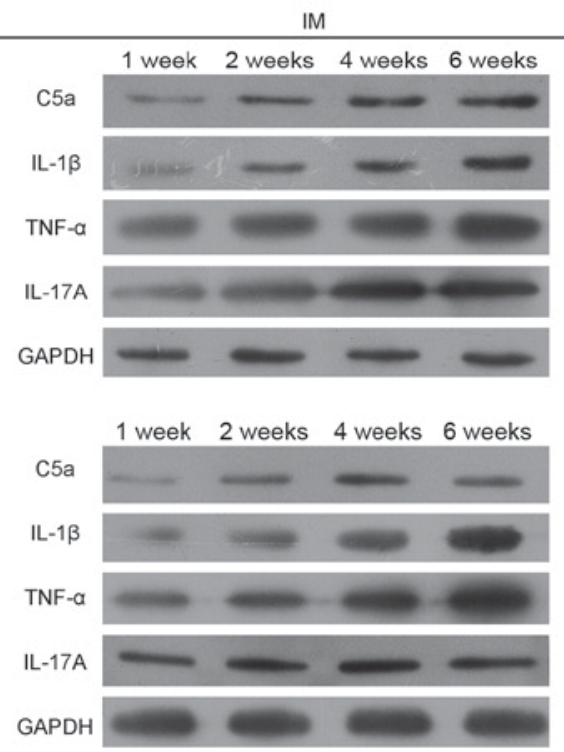

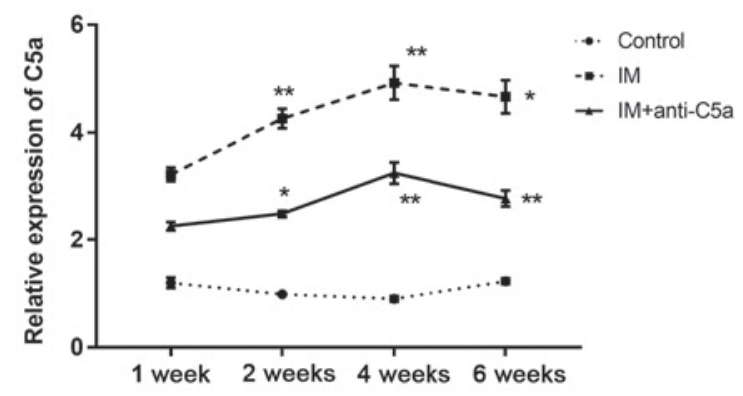

IM+anti-C5a
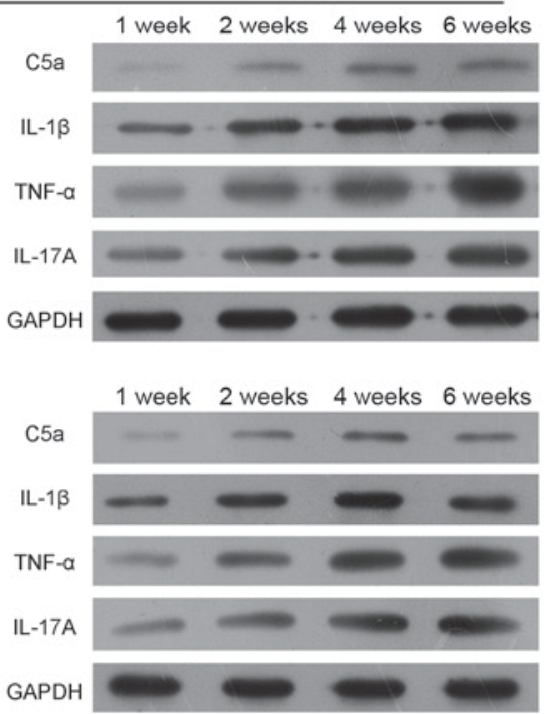

Figure 6. Expression levels of C5a in the synovialis and cartilage of rats with knee joint IM at different time points. (A) C5a mRNA expression; (B) Representative bands of C5a protein in the synovialis of rats at different time points; (C) Representative bands of C5a protein expression in the cartilage at different time points. The graphs illustrate the mean \pm SEM. ${ }^{*} \mathrm{P}<0.05,{ }^{* *} \mathrm{P}<0.01$ vs. control group at the same time point. The data are presented as the average of triplicate values. Error bars represent the SEM. IM, immobilization; C5a, complement component 5a; SEM, standard error of the mean; IL, interleukin; TNF- $\alpha$, tumor necrosis factor- $\alpha$.

OA. The levels of a number of pro-inflammatory cytokines, including IL-1 $\beta$, TNF- $\alpha$ and IL-17A, were significantly increased during the experiment, and the present results provided novel insights into the hypothesis.

In the present study, joint IM rat models were used. Joint IM has been reported to cause degeneration or atrophy of the articular cartilage (36-38). Loading and movement remains important for the maintenance of morphological and functional integrity of the joint articular cartilage (7,39). Immobilization was able to induce increased hydration, decreased proteoglycan content, altered PG aggregate structure and increased collagen synthesis, and was able to maintain or elevate the collagen content of the articular cartilage $(2,11,38)$. Furthermore, following joint IM, destruction of the knee joint synovial fluid and cartilage was observed. Masson staining revealed increased collagen in the synovial fluid and cartilage, in addition to alterations in pro-inflammatory cytokine expression. These results suggested that the joint IM model was a suitable animal model for OA.

In conclusion, the present study investigated the role of the C5a pathway in the development of OA, and demonstrated that the expression of C5a was increased in the synoviocytes and cartilage in $\mathrm{OA}$ joint diseases. Furthermore, the release of a number of pro-inflammatory cytokines, including TNF- $\alpha$ and IL-1 $\beta$, was induced in the activated synoviocytes via interactions with C5a, suggesting that the C5a system may serve an important role in joint inflammatory processes.

\section{Acknowledgements}

Not applicable.

\section{Funding}

The present was supported by Science and Technology Cooperation Program of Guizhou Province [GZSC LH (2015). no. 7376], Science Program of Guizhou Province [GZSS SY (2015). no. 3043] and the Science and Technology Bureau of Guiyang [(2017). no. 30-35).

\section{Availability of data and materials}

All data generated or analyzed during this study are included in this published article.

\section{Authors' contributions}

WL and LW conceptualized the study design. WL, LW and JY contributed to data analyses. WL and JY prepared the manuscript. WL, CW and YC performed the data analyses and 
wrote the manuscript. All authors read and approved the final manuscript.

\section{Ethics approval and consent to participate}

All procedures were approved by the Guizhou Medical University (Guiyang, China) Animal Care and Use Committees.

\section{Patient consent for publication}

Not applicable.

\section{Competing interests}

The authors declare that they have no competing interests.

\section{References}

1. Behrens F, Kraft EL and Oegema TR Jr: Biochemical changes in articular cartilage after joint immobilization by casting or external fixation. J Orthop Res 7: 335-343, 1989.

2. Tammi M, Säämänen AM, Jauhiainen A, Malminen $O$, Kiviranta I and Helminen H: Proteoglycan alterations in rabbit knee articular cartilage following physical exercise and immobilization. Connect Tissue Res 11: 45-55, 1983.

3. Tammi M, Kiviranta I, Peltonen L, Jurvelin J and Helminen HJ: Effects of joint loading on articular cartilage collagen metabolism: Assay of procollagen prolyl 4-hydroxylase and galactosylhydroxylysyl glucosyltransferase. Connect Tissue Res 17: 199-206, 1988.

4. Videman T, Eronen I and Candolin T: [3H]proline incorporation and hydroxyproline concentration in articular cartilage during the development of osteoarthritis caused by immobilization. A study in vivo with rabbits. Biochem J 200: 435-440, 1981.

5. Jurvelin J, Kiviranta I, Säämänen AM, Tammi M and Helminen HJ: Partial restoration of immobilization-induced softening of canine articular cartilage after remobilization of the knee (stifle) joint. J Orthop Res 7: 352-358, 1989.

6. Haapala J, Arokoski JP, Hyttinen MM, Lammi M, Tammi M, Kovanen V, Helminen HJ and Kiviranta I: Remobilization does not fully restore immobilization induced articular cartilage atrophy. Clin Orthop Relat Res: 218-229, 1999.

7. Jortikka MO, Inkinen RI, Tammi MI, Parkkinen JJ, Haapala J, Kiviranta I, Helminen HJ and Lammi MJ: Immobilisation causes longlasting matrix changes both in the immobilised and contralateral joint cartilage. Ann Rheum Dis 56: 255-261, 1997.

8. Vanwanseele B, Eckstein F, Knecht H, Stussi E and Spaepen A: Knee cartilage of spinal cord-injured patients displays progressive thinning in the absence of normal joint loading and movement. Arthritis Rheum 46: 2073-2078, 2002.

9. Hagiwara Y, Ando A, Chimoto E, Saijo Y, Ohmori-Matsuda K and Itoi E: Changes of articular cartilage after immobilization in a rat knee contracture model. J Orthop Res 27: 236-242, 2009.

10. Pritzker KP: Animal models for osteoarthritis: Processes, problems and prospects. Ann Rheum Dis 53: 406-420, 1994.

11. Wong K, Sun F, Trudel G, Sebastiani P and Laneuville O: Temporal gene expression profiling of the rat knee joint capsule during immobilization-induced joint contractures. BMC Musculoskelet Disord 16: 125, 2015.

12. Misra D, Felson DT, Silliman RA, Nevitt M, Lewis CE, Torner J and Neogi T: Knee osteoarthritis and frailty: Findings from the Multicenter Osteoarthritis Study and Osteoarthritis Initiative. J Gerontol A Biol Sci Med Sci 70: 339-344, 2015.

13. Goldring MB and Goldring SR: Articular cartilage and subchondral bone in the pathogenesis of osteoarthritis. Ann N Y Acad Sci 1192: 230-237, 2010.

14. Haseeb A and Haqqi TM: Immunopathogenesis of osteoarthritis. Clin Immunol 146: 185-196, 2013.

15. Berenbaum F: Osteoarthritis as an inflammatory disease (osteoarthritis is not osteoarthrosis!). Osteoarthritis Cartilage 21: 16-21, 2013.

16. Liu-Bryan R: Synovium and the innate inflammatory network in osteoarthritis progression. Curr Rheumatol Rep 15: 323, 2013.

17. Nishiura H: The alternative C5a receptor function. Adv Exp Med Biol 735: 111-121, 2013
18. Woodruff TM, Nandakumar KS and Tedesco F: Inhibiting the C5-C5a receptor axis. Mol Immunol 48: 1631-1642, 2011.

19. Yuan G, Wei J, Zhou J, Hu H, Tang Z and Zhang G: Expression of C5aR (CD88) of synoviocytes isolated from patients with rheumatoid arthritis and osteoarthritis. Chin Med J (Engl) 116: 1408-1412, 2003.

20. Pujol JP and Loyau G: Interleukin-1 and osteoarthritis. Life Sci 41: 1187-1198, 1987.

21. National Institute of Allergy and Infectious Diseases NIoH, Bethesda, Md: National Institutes of Health (1996) Guide for the care and use of laboratory animals.

22. Livak KJ and Schmittgen TD: Analysis of relative gene expression data using real-time quantitative PCR and the 2(-Delta Delta C(T)) method. Methods 25: 402-408, 2001.

23. Ponchel F, Burska AN, Hensor EM, Raja R, Campbell M, Emery P and Conaghan PG: Changes in peripheral blood immune cell composition in osteoarthritis. Osteoarthritis Cartilage 23: 1870-1878, 2015

24. Nykula TD, Bychkov OA and Bychkova NH: The dynamics of indices of immune and cytokine status for patients with essential hypertension in combination with osteoarthritis. Lik Sprava: 90-93, 2013 (In Ukrainian).

25. Kandahari AM, Yang X, Dighe AS, Pan D and Cui Q: Recognition of immune response for the early diagnosis and treatment of osteoarthritis. J Immunol Res 2015: 192415, 2015.

26. de Lange-Brokaar BJ,Ioan-Facsinay A, van Osch GJ,Zuurmond AM, Schoones J, Toes RE, Huizinga TW and Kloppenburg M: Synovial inflammation, immune cells and their cytokines in osteoarthritis: A review. Osteoarthritis Cartilage 20: 1484-1499, 2012.

27. Wang Q, Rozelle AL, Lepus CM, Scanzello CR, Song JJ, Larsen DM, Crish JF, Bebek G, Ritter SY, Lindstrom TM, et al: Identification of a central role for complement in osteoarthritis. Nat Med 17: 1674-1679, 2011.

28. Buckland J: Osteoarthritis: Complement-mediated inflammation in OA progression. Nat Rev Rheumatol 8: 2, 2011.

29. Liu-Bryan R and Terkeltaub R: Emerging regulators of the inflammatory process in osteoarthritis. Nat Rev Rheumatol 11: 35-44, 2015.

30. Struglics A, Okroj M, Swärd P, Frobell R, Saxne T, Lohmander LS and Blom AM: The complement system is activated in synovial fluid from subjects with knee injury and from patients with osteoarthritis. Arthritis Res Ther 18: 223, 2016.

31. Wang X, Hunter D, Xu J and Ding C: Metabolic triggered inflammation in osteoarthritis. Osteoarthritis Cartilage 23: 22-30, 2015.

32. Kuna P, Reddigari SR, Schall TJ, Rucinski D, Sadick M and Kaplan AP: Characterization of the human basophil response to cytokines, growth factors, and histamine releasing factors of the intercrine/chemokine family. J Immunol 150: 1932-1943, 1993.

33. Stone SF, Isbister GK, Shahmy S, Mohamed F, Abeysinghe C, Karunathilake H, Ariaratnam A, Jacoby-Alner TE, Cotterell CL and Brown SG: Immune response to snake envenoming and treatment with antivenom; complement activation, cytokine production and mast cell degranulation. PLoS Negl Trop Dis 7: e2326, 2013.

34. Michel O, Ginanni R, Le Bon B, Content J, Duchateau J and Sergysels R: Inflammatory response to acute inhalation of endotoxin in asthmatic patients. Am Rev Respir Dis 146: 352-357, 1992.

35. Cavaillon JM, Fitting C and Haeffner-Cavaillon N: Recombinant C5a enhances interleukin 1 and tumor necrosis factor release by lipopolysaccharide-stimulated monocytes and macrophages. Eur J Immunol 20: 253-257, 1990.

36. Kiviranta I, Tammi M, Jurvelin J, Arokoski J, Saamanen AM and Helminen HJ: Articular cartilage thickness and glycosaminoglycan distribution in the young canine knee joint after remobilization of the immobilized limb. J Orthop Res 12: 161-167, 1994.

37. Maldonado DC, Silva MC, Neto Sel-R, de Souza MR and de Souza RR: The effects of joint immobilization on articular cartilage of the knee in previously exercised rats. J Anat 222: 518-525, 2013.

38. Nomura M, Sakitani N, Iwasawa H, Kohara Y, Takano S, Wakimoto Y, Kuroki $\mathrm{H}$ and Moriyama H: Thinning of articular cartilage after joint unloading or immobilization. An experimental investigation of the pathogenesis in mice. Osteoarthritis Cartilage 25: 727-736, 2017.

39. Müller FJ, Setton LA, Manicourt DH, Mow VC, Howell DS and Pita JC: Centrifugal and biochemical comparison of proteoglycan aggregates from articular cartilage in experimental joint disuse and joint instability. J Orthop Res 12: 498-508, 1994. 